\title{
Avaliação dos valores gasométricos em cavalos (Equus caballus) da raça Puro-Sangue-Inglês (PSI) submetidos a teste de esforço em esteira ergométrica*
}

\section{Evaluation of hemogasometric values in Thoroughbred horses (Equus caballus) submitted to effort tests on treadmill}

\author{
Rosalie Joslin Kowal, ${ }^{* *}$ Bianca Cascardo, ${ }^{* * *}$ Ricardo Peixoto Summa, ${ }^{\star * *}$ Leopoldo José Cury, ${ }^{* \star * *}$ \\ Jorge Morgado Neto, ${ }^{* * * *}$ Nádia Regina Pereira Almosny******
}

\begin{abstract}
Resumo
O Puro-Sangue-Inglês (PSI), raça nobre de origem inglesa especializada em corridas, encontra-se difundido em todo o Brasil e, dada a falta de dados sobre a fisiologia do exercício destes animais torna-se importante a realização de testes de esforço sob algumas condições características visando a obter informações mais precisas e adequadas quando esses cavalos são submetidos a esforços constantes. Alguns parâmetros mostram-se de grande valia na determinação de performance. Foram utilizados 20 animais em fase de treinamento no Jockey Club Brasileiro, com idade entre 2 e 6 anos, machos e fêmeas,

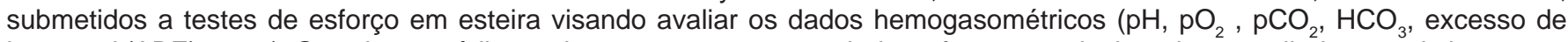
base real (ABE) e p50). Os valores médios variaram nos tempos estudados e foram correlacionados e avaliados estatisticamente. Concluiu-se que a realização de teste de esforço em esteira ergométrica é útil para a determinação destes parâmetros e tem como principal função avaliar a capacidade atlética de cavalos de corridas, bem como detectar possíveis momentos de baixa performance permitindo a sua correção.
\end{abstract}

Palavras-chave: eqüino, esteira, exercício, hemogasometria.

\begin{abstract}
Thoroughbred horses, a noble English breed specialized in races, are spread in Brazil and due to the lack of data concerning their exercise physiology, it is important to do exercise tests under some characteristic conditions in order to obtain precise and proper data as these horses are submitted to constant efforts. Twenty Thoroughbred racehorses in training at Jockey Club Brasileiro, with age ranging from 2 to 6 years old, males and females, submitted to effort tests on treadmill in order to evaluate hemogasometric $\left(\mathrm{pH}, \mathrm{pO}_{2}, \mathrm{pCO}_{2}, \mathrm{HCO}_{3}\right.$, actual base excess (ABE) and $\left.\mathrm{p50}\right)$ values. The mean values varied at the studied times and they were statistically evaluated and correlated. It was concluded that the exercise tests on a treadmill are useful for the determination of this parameters and they have as main role to evaluate the athletic ability of racehorses, as well as to detect possible poor performance times, allowing their correction.
\end{abstract}

Keywords: equine, treadmill, exercise, blood.

\section{Introdução}

O cavalo (Equus caballus) encontra-se hoje amplamente difundido no mundo servindo ao homem não só para o lazer, mas também nos esportes e, dentre todos os que os envolvem, o turfe tem papel econômico de marcada importância.

A hemogasometria está relacionada com a análise do $\mathrm{pH}$, dos gases sanguíneos e seus derivados gasosos, os quais podem apresentar variações decorrentes de diferentes causas, sejam elas respiratórias e/ou metabólicas (Rose e Hodgson, 1994).
Parte dos distúrbios ácido-básicos encontrados no cavalo são devido à prática de exercícios, sendo que a duração desse distúrbio está diretamente relacionada com a intensidade da atividade a que o animal é submetido (Rose, s.d.).

Nos exercícios de alta intensidade a atividade respiratória, o movimento livre de água do compartimento intravascular e alterações na temperatura sangüínea afetam o equilíbrio ácidobásico (Constable, 1999). Significante acidose metabólica se desenvolve em cavalos em exercício (Manohar et al., 2001).

\footnotetext{
* Trabalho de tese de mestrado. Universidade Federal Fluminense.

** Médica-veterinária. MSc. Jockey Club Brasileiro. Rio de Janeiro.

*** Médicos-veterinários. Jockey Club Brasileiro. Rio de Janeiro.

**** Médico-veterinário. MSc. Jockey Club Brasileiro. Rio de Janeiro. Universidade Federal Fluminense.

***** Médico-veterinário Residente. Jockey Club Brasileiro. Rio de Janeiro.

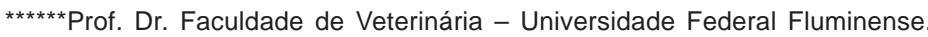


No exercício intenso a temperatura aumenta de $37^{\circ} \mathrm{C}$ para $41^{\circ} \mathrm{C}$ e isso leva a redução do $\mathrm{pH}$ de aproximadamente 0,06 (Constable, 1999).

Define-se acidose metabólica como a queda dos níveis plasmáticos de íons bicarbonato, conseqüente à sua eliminação acima do normal, ou então, pelo acréscimo de elementos ácidos ao organismo, ou ainda, pela diminuição da "clearance" renal de ácidos fixos (Castro et al., 1994).

A acidose consequente aos exercícios de altas intensidades costuma ser mais intensa nos primeiros cinco minutos após o término da atividade. Considerando-se que o metabolismo do lactato varia de cavalo para cavalo, muitos deles costumam restabelecer a normalidade do equilíbrio ácido-básico na primeira hora após o exercício. Em razão dessa rapidez metabólica, não é usual constatar-se estados de acidose metabólica mantida em animais de elevada capacidade atlética (Rose, s.d.).

Os cavalos, quando submetidos a exercícios de alta intensidade, desenvolvem metabolismo oxidativo gerador de íons $\mathrm{H}^{+}$responsáveis pela produção endógena de ácido. Esta ocorrência determina queda do $\mathrm{pH}$ que provocará estímulo do centro respiratório, seja por intermédio de quimiorreceptores periféricos, seja através de alterações do pH do líquor e do interstício cerebral, beneficiando a oxigenação tissular em razão de liberação mais rápida do oxigênio pela hemoglobina, ou seja, menor afinidade da hemoglobina com o oxigênio, segundo Castro et al., (1994).

A acidemia transitória estimula a produção de catecolaminas, com conseqüente elevação da pressão arterial e aumento da freqüência cardíaca (taquicardia) (Castro et al., 1994).

A tendência de queda da $\mathrm{pCO}_{2}$ quando a ventilação alveolar aumenta (taquipnéia) contrapõ̃e e supera $\mathrm{C} \mathrm{CO}_{2}$ produzido pelo metabolismo celular. Essa condição de hiperventilação ocorre na acidose metabólica com o propósito de ajustar o $\mathrm{pH}$ ao seu nível de normalidade, em menor intensidade durante o exercício (Trim, 1999).

Nos cavalos, a grande área de superfície alveolar por kg de peso corporal satisfaz o grande consumo de oxigênio obtido durante o exercício. Quando o sangue contendo grande quantidade de hemoglobina não-saturada passa através dos pulmões em alta velocidade, ou seja, quando o tempo de trânsito do sangue pelos capilares pulmonares é rápido e o débito cardíaco está aumentado, como costuma ocorrer durante os exercícios intensos, o tempo pode ser insuficiente para estabelecer equilíbrio nas tensões de $\mathrm{O}_{2}$ alveolar e sanguínea. Essa é a causa mais provável de hipoxemia observada nos cavalos em exercício. Os animais apresentarão hipoventilação relativa evidenciada pela $\mathrm{pCO}_{2}$ aumentada. Entretanto, deve-se considerar que a extração de oxigênio pelos músculos está aumentada (Christley et al., 1999; Roberts et al., 1999; Trim, 1999; Manohar et al., 2001).

Acredita-se que na falta de adaptação pulmonar ao exercício se desenvolva edema perivascular no tecido pulmonar, possivelmente limitando a difusão do oxigênio e favorecendo a redução da performance (Roberts et al., 1999; Manohar et al., 2001).

A raça especializada em corridas, o Puro-Sangue-Inglês (PSI), é encontrada em todo território nacional e, dada a falta de dados sobre a fisiologia do exercício destes animais em nosso país, torna-se importante a realização de testes de esforço para obter informações mais precisas e adequadas dos nossos cavalos quando submetidos a esforços constantes.

Logo, o objetivo deste experimento é coletar dados hemogasométricos de cavalos de corridas submetidos a teste de esforço e investigar as causas de baixa capacidade atlética utilizando-se esteira ergométrica.

\section{Material e métodos}

Foram utilizados neste trabalho 20 animais da raça PuroSangue-Inglês (PSI), na faixa etária de 2 a 6 anos, machos e fêmeas, em fase de treinamento no Jockey Club Brasileiro. Esse treinamento é de responsabilidade do treinador do animal, sendo por ele determinado.

Em todos os animais foram realizados exames clínicos rotineiros, em repouso, e avaliação do histórico do seu desempenho atlético. Todos os animais apresentaram boas condições físicas, sem quaisquer problemas aparentes. Os animais foram submetidos a um período de adaptação ao exercício na esteira ergométrica GALLOPER 5500. O programa de adaptação levou de cinco a sete dias, consistindo desde o simples reconhecimento do animal à esteira até o galope confortável. Nesta fase foram registrados o batimento cardíaco e o comportamento dos animais nas diferentes velocidades a que foram submetidos. O programa de adaptação foi modificado ou ajustado às peculiaridades de cada animal.

O seguinte esquema foi adotado:

Inclinação de 6 graus, em relação ao solo, na esteira: 4 minutos a $2 \mathrm{~m} / \mathrm{s}-3$ minutos a $4 \mathrm{~m} / \mathrm{s}-2$ minutos a $6 \mathrm{~m} / \mathrm{s}-1$ minuto a $8 \mathrm{~m} / \mathrm{s}-3$ minutos a $4 \mathrm{~m} / \mathrm{s}-1$ minuto a $2 \mathrm{~m} / \mathrm{s}$. Uma vez estando o animal apto a realizar o teste de esforço seguiuse o procedimento adiante descrito:

Foram realizadas coletas de amostras de sangue na cocheira, com o animal em repouso, em seringa ${ }^{1}$ de $3 \mathrm{~mL}$ previamente heparinizada ${ }^{2}$ para análise gasométrica, com cuidado para não aspirar bolhas de ar, que poderiam alterar o resultado da análise. As amostras eram imediatamente armazenadas em recipiente isotérmico e levadas em seguida ao laboratório do Hospital Octávio Dupont onde eram realizadas as análises.

1) $O$ animal então era conduzido à esteira ergométrica, davase a inclinação de 6 graus, para simular condições similares àquelas estabelecidas em condições de corridas, como compensar a ausência do peso do jóquei, e dava-se início ao exercício.

2) O exercício dava-se da seguinte forma: 2 minutos a $2 \mathrm{~m} / \mathrm{s}, 4$ minutos a $3 \mathrm{~m} / \mathrm{s}, 1$ minuto a $5 \mathrm{~m} / \mathrm{s}, 1$ minuto a $6 \mathrm{~m} / \mathrm{s}$ e assim, sucessivamente até a máxima velocidade capaz de ser desenvolvida pelo animal.

3) No momento correspondente ao término do exercício, uma nova amostra de sangue era coletada em seringa heparinizada, da veia jugular esquerda, para análise hemogasométrica.

4) A gasometria venosa consistiu das seguintes análises: $\mathrm{pH}, \mathrm{pCO}_{2}, \mathrm{pO}_{2}, \mathrm{HCO}_{3}, \mathrm{ABE}$ e $\mathrm{P} 50$, e foram realizadas no aparelho ABL 530 da Radiometer.

Em todos os momentos acompanhados foram observadas e anotadas as freqüências cardíacas dos animais, sendo impor-

\footnotetext{
1 Seringas de $3 \mathrm{~mL}$ BD Plastipak

2 Heparina sódica - Liquemine - Roche - 25000UI
} 
tante para este trabalho as frequências nos instantes finais do teste de esforço, para determinação do seu término. A mínima freqüência observada foi de 205 bpm e a máxima foi de 237 bpm.

O tempo de permanência do animal na esteira durante o teste de esforço variou de 14 a 18 minutos, dependendo da velocidade atingida pelo animal no seu máximo esforço físico.

\section{Delineamento estatístico}

Os dados laboratoriais foram analisados estatisticamente por método não-paramétrico de Wilcoxon com nível de significância de 5\% (Rodrigues, 1993).

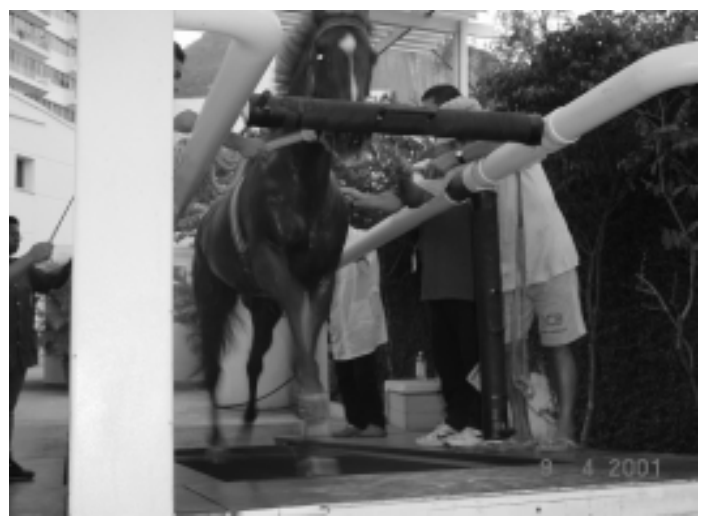

Figura 1: Animal em galope $(8 \mathrm{~m} / \mathrm{s})$ em esteira ergométrica durante $o$ teste de esforço.

\section{Resultados e discussão}

Tabela 1: Valores de gasometria antes do exercício de cavalos submetidos a teste de esforço em esteira ergométrica

\begin{tabular}{l|ccccc}
\hline & $\mathrm{n}$ & Média & Desvio-padrão & Valor mínimo & Valor máximo \\
\cline { 2 - 6 } Temperatura & 19 & 37,5 & 3,1 & 37,5 & 37,6 \\
Hemoglobina & 19 & 14,4 & 1,1 & 12,7 & 17,0 \\
$\mathrm{pH}$ & 19 & 7,34 & 0,06 & 7,14 & 7,39 \\
$\mathrm{pCO}_{2}$ & 19 & 56,08 & 8,08 & 48,8 & 87,7 \\
$\mathrm{pO}_{2}$ & 19 & 38,71 & 7,40 & 29,60 & 62,20 \\
$\mathrm{HCO}_{3}$ & 19 & 29,90 & 2,92 & 23,00 & 34,90 \\
$\mathrm{ABE}$ & 19 & 3,18 & 3,22 & $-4,10$ & 8,20 \\
$\mathrm{P50}$ & 19 & 23,50 & 0,00 & 23,50 & 23,50 \\
\hline
\end{tabular}

T: temperatura $\left({ }^{\circ} \mathrm{C}\right) ; \mathrm{Hb}$ : concentração de hemoglobina $(\mathrm{g} / \mathrm{dL}) ; \mathrm{pH}$ : potencial hidrogeniônico; $\mathrm{pCO}_{2}$ : pressão de $\mathrm{CO}_{2} ; \mathrm{pO}_{2}$ : pressão de $\mathrm{O}_{2} ; \mathrm{HCO}_{3}$ : concentração de bicarbonato; $\mathrm{ABE}$ : excesso de base real; P50: tensão de $\mathrm{O}_{2}$ onde $50 \%$ da hemoglobina encontra-se saturada.

Tabela 2: Valores de gasometria depois do exercício de equinos submetidos a teste de esforço em esteira ergométrica

\begin{tabular}{l|ccccc}
\hline & $\mathrm{n}$ & Média & Desvio-padrão & Valor mínimo & Valor máximo \\
\cline { 2 - 6 } Temperatura & 19 & 39,2 & 0,3 & 38,5 & 39,5 \\
Hemoglobina & 19 & 21,5 & 1,1 & 19,1 & 23,7 \\
$\mathrm{pH}$ & 19 & 7,17 & 0,8 & 7,01 & 7,27 \\
$\mathrm{pCO}_{2}$ & 19 & 40,48 & 9,06 & 27,30 & 56,80 \\
$\mathrm{PO}_{2}$ & 19 & 56,70 & 7,34 & 44,90 & 70,10 \\
$\mathrm{HCO}_{3}$ & 19 & 14,13 & 4,41 & 8,00 & 21,80 \\
$\mathrm{ABE}$ & 19 & $-14,23$ & 6,06 & $-24,50$ & $-5,50$ \\
$\mathrm{P50}$ & 19 & 23,50 & 0,00 & 23,50 & 23,50 \\
\hline
\end{tabular}

$\mathrm{T}$ : temperatura $\left({ }^{\circ} \mathrm{C}\right) ; \mathrm{Hb}$ : concentração de hemoglobina $(\mathrm{g} / \mathrm{dL}) ; \mathrm{pH}$ : potencial hidrogeniônico; $\mathrm{pCO}_{2}$ : pressão de $\mathrm{CO}_{2} ; \mathrm{pO}_{2}$ : pressão de $\mathrm{O}_{2} ; \mathrm{HCO}_{3}$ : concentração de bicarbonato; ABE: excesso de base real; P50: tensão de $\mathrm{O}_{2}$ onde $50 \%$ da hemoglobina encontra-se saturada.
Os valores referentes à análise gasométrica apresentaram rença significativa ( $p$ valor $<0,05$ ) para todos os valores Os valores de $\mathrm{pH}$ decrescem imediatamente após o término exercício, acompanhado de elevação da $\mathrm{pO}_{2}$ e redução da (1999), no ińcio de músculo é tão alta que leva a acidose, com o $\mathrm{pH}$ podendo chegar a 6,5.

experimento realizado por Schuback et al. (1999), a fadiga diferentes velocidades $(9-11 \mathrm{~m} / \mathrm{s})$ e a temperatura ocor-reu 35,9 a $41,4 C$. No pre-sente experimento a fadiga variaram entre 37,5 e $39,2 \mathrm{C}^{\circ}$. A fadiga muscular ocorre como mecanismo de defesa do organismo, devido à elevada Os dados deste experimento indicaram que o condicionamento físico reduz a queda de $\mathrm{pH}$, conforme também afirmaram Snow e Mackenzie (1977) e Constable (1999).

e Mackenzie (1977) relataram que a $\mathrm{pO}_{2}$ aumenta e que a $\mathrm{HCO}_{3}$ diminuem com o exercício. $\mathrm{O} \mathrm{pH}$ apresenta a, mas com o treinamento essa queda tende a ser menor. (2001), com o aumento da intensidade aumento da do sangue arterial diminui até 7,12 em $14 \mathrm{~m} / \mathrm{s}$, indicando a

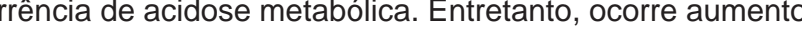
do conteúdo arterial de $\mathrm{O}_{2}$ durante o exercício, principalmente devido ao aumento de $50 \%$ na concentração de hemoglobina resultante da liberação de hemácias da reserva esplênica para a circulação.

Christley et al. (1999), entretanto, observaram em seu estudo que existe uma cinética bastante rápida das funções metabólica e respiratória do cavalo em exercício de rápida aceleração. Eles observaram diminuição da $\mathrm{pO}_{2}$ e aumento da $\mathrm{pCO}_{2}$ e relataram que o conteúdo arterial de oxigênio foi mantido apesar de haver redução na saturação da hemoglobina. Em estudo realizado por Roberts et al. (1999), entretanto, onde foram avaliados os efeitos do treinamento sobre os gases sanguíneos, verificou-se que ${\mathrm{a} \mathrm{pO}_{2}}_{2}$ aumenta depois da fase de treinamento, enquanto que a $\mathrm{pCO}_{2}$ não se altera. No presente experimento observou-se elevação de $\mathrm{pO}_{2}$ e redução da $\mathrm{pCO}_{2}$ e estes dados foram justificados pela resposta respiratória decorrente da elevação dos níveis de lactato relatados por Valette e Auvinet (1993), Rainger et al. (1995), Geor et al. (1999), Fenger et al. (2000) e Manohar et al. (2001). Segundo Mayes (1998) e Fenger et al. (2000) a acidose metabólica determina a redução da afinidade da hemoglobina pelo $\mathrm{CO}_{2}$ com conseqüente resposta hiperventilatória por parte do animal.

As freqüências cardíacas dos animais utilizados neste experimento foram anotadas desde o momento repouso até o momento em que se aproximavam dos valores basais, 
em até 30 minutos após o término do exercício. Nos momentos finais do teste de esforço a FC (freqüência cardíaca) atingiu os valores de 220 a 240 bpm. A partir do instante em que essas FC eram observadas o teste poderia terminar a qualquer momento, ficando a critério da equipe envolvida na realização do teste determinar o momento de reduzir a velocidade da esteira, quando o animal apresentava os primeiros sinais de fadiga muscular.

\section{Conclusão e sugestões}

O teste de esforço em esteira ergométrica permite a determinação de valores hemogasométricos, valores esses de extrema importância na avaliação da capacidade atlética desejável. Tal capacidade é compreendida como a integração dos vários sistemas corporais, os quais determinam a produção de energia despendida em uma disputa.

\section{Referências}

CASTRO, A. C.; HUEHARA, M.; VIEIRA, C. C. Equilíbrio Ácido-Básico. In: CASTRO, Antônio C.; HUEHARA, M.; VIEIRA, Celina C. (Ed.) Propedêutica do Equilíbrio hidroeletrolítico e ácido-básico. Rio de Janeiro: Atheneu, 1994. cap. 4. p. 51-94.

CHRISTLEY, R. M.; EVANS, D. L.; HODGSON, D. R.; ROSE, R. J. Blood Gas Changes During Incremental and Sprint Exercise. Equine Exercise Phisiology (5), Equine Veterinary Journal. Suppl. 30, p. 24-26, 1999.

CONSTABLE, P. D. The Physicochemical Approach for Evaluating AcidBase Balance in Exercising Horses. Equine Exercise Physiology 5. Equine Veterinary Journal. Suppl. 30, p. 636-638, 1999.

FENGER, C. K.; McKEEVER, K. H.; HINCHCLIFF, K. W.; KOHN, C. W. Determinants of Oxygen Delivery and Hemoglobin Saturation During Incremental Exercise in Horses. American Journal of Veterinary Research. v. 61, n. 10, p. 1325-1332, out, 2000.

GEOR, R. J.; McCUTCHEON, L. J.; SHEN, H. Muscular and Metabolic Responses to Moderate-Intensity Short-Term Training. Equine Exercise Physiology 5. Equine Veterinary Journal. Suppl. 30, p. 311-317, 1999. MANOHAR, M.; GOETZ, T. E.; HASSAN, A. S. Effect of Prior HighIntensity Exercise-Induced Arterial Hypoxemia in Thoroughbred Horses. Journal of Applied Physiology. v. 90, n. 6, p. 2371-2377, Jun., 2001.

MAYES, P. A. Bioenergética e metabolismo de carboidratos e lipídeos. In: MURRAY, R. K.; GRANNER, D. K.; MAYES, P. A.; RODWELL, V.W. Harper: Bioquímica. 8. ed. São Paulo: Atheneu, 1998. Seção II, cap. 12, p. 109-291. RAINGER. J. E.; EVANS, D. L.; HODGSON, D. R.; ROSE, R. J. Distribution of Lactate in Plasma and Erythrocytes During and After Exercise in Horses. British Veterinary Journal, 151, p. 299-310, 1995.
Com o aumento da intensidade do exercício os animais passam a apresentar sinais de leve acidose metabólica.

As velocidades desenvolvidas na esteira podem ser semeIhantes àquelas a que os animais desenvolvem nas corridas. Isto equivale a dizer que na esteira é possível desenvolver velocidade de rápida aceleração, a partir do momento em que está em inércia até uma velocidade de aproximadamente 15 $\mathrm{m} / \mathrm{s}$, para que seja possível simular condições similares àquelas estabelecidas em condições de corrida.

Pode-se recomendar como válido o teste em esteira ergométrica, com a finalidade de padronizar os valores hemogasométricos nos cavalos de corridas, com vistas a uma avaliação mais confiável do seu condicionamento físico, levandose ainda em consideração o fato de que o controle do monitoramento do animal costuma ser seguro e confiável durante as diferentes fases do teste.

ROBERTS, C.A.; MARLIN, D.J.; LEKEUX, P. The Effects of Training on Ventilation and Blood Gases in Exercising Thoroughbreds. Equine Exercise Physiology 5. Equine Veterinary Journal. Suppl. 30, p. 57-61, 1999.

RODRIGUES, P. C. Teste de Hipóteses. In: Bioestatística. 2. ed. Niterói: EdUFF, 1993. cap. 12, p. 79-88.

ROSE, R.J. Problems of the Performance and Endurance Horse - Fluid, Electrolyte and Acid-Base Disturbances Associated with Exercise. In: ROBINSON, E. (ed.) Current Therapy in Equine Medicine 2. W. B. Saunders Company. seção 12, p. 479-481.

ROSE, R. J.; HODGSOND. R. Structural Considerations in Equine Sports Medicine-Hematology and Biochemistry. In: The Athletic Horse. W. B. Saunders Company, 1994. 497 p., seção 1, cap. 5, p. 63-78.

SCHUBACK, K; ESSÉN-GUSTAVSSON, B.; PERSSON, G.B. Incremental Treadmill Exercise Until Onset of Fatigue and its Relationship to Metabolic Response and Locomotion Pattern. Equine Exercise Phisiology (5), Equine Veterinary Journal. Suppl. 30, p. 337-341, 1999.

SNOW, D.H.; MACKENZIE, G. Some Metabolic Effects of Maximal Exercise in the Horse and Adaptations with Training. Equine Veterinary Journal. v. 9, n. 3, p. 134-140, 1977.

TRIM, C.M. Monitoring Inhalation Anesthesia. In: COLAHAN, P. T.; MAYHEW, I. G.; MERRIT, A. M.; MOORE, J. N. Equine Medicine \& Surgery. 5. ed. Mosby, 1999. p. 268-272.

VALETTE, J. P.; BARREY, E.; AUVINET, B.; GALLOUX, P.; WOLTER, R. Exercise Tests in Saddle Horses. 2: The Kinetics of Blood Lactate During Constant Exercise Tests on a Treadmill. Journal of Equine Veterinary Science. v. 13, n. 8, p. 465-468, 1993. 